\title{
Existence of Solutions for a Periodic Boundary Value Problem with Impulse and Fractional Derivative Dependence
}

\author{
Yaohong Li $\mathbb{D}$, ${ }^{1}$ Yongqing Wang $\mathbb{D},{ }^{2}$ Donal O'Regan $\left(\mathbb{D},{ }^{3}\right.$ and Jiafa Xu $\mathbb{C D}^{4}$ \\ ${ }^{1}$ School of Mathematics and Statistics, Suzhou University, Suzhou 234000, Anhui, China \\ ${ }^{2}$ School of Mathematical Sciences, Qufu Normal University, Qufu, Shandong 273165, China \\ ${ }^{3}$ School of Mathematics, Statistics and Applied Mathematics, National University of Ireland Galway, Galway, Ireland \\ ${ }^{4}$ School of Mathematical Sciences, Chongqing Normal University, Chongqing 401331, China \\ Correspondence should be addressed to Yongqing Wang; wyqing9801@163.com
}

Received 10 October 2020; Revised 6 December 2020; Accepted 9 December 2020; Published 29 December 2020

Academic Editor: Xiaolong Qin

Copyright ( $\odot 2020$ Yaohong Li et al. This is an open access article distributed under the Creative Commons Attribution License, which permits unrestricted use, distribution, and reproduction in any medium, provided the original work is properly cited.

In this paper, we present some theorems on impulsive periodic boundary value problems with fractional derivative dependence. In particular, we discuss the existence of solutions of a class of fractional-order impulsive periodic boundary values with nonlinear terms and impulsive terms satisfying certain growth conditions. Three examples are provided to illustrate our results.

\section{Introduction}

This paper considers the existence of solutions of the following fractional-order impulsive periodic boundary value problem:

$$
\left\{\begin{array}{l}
{ }^{c} D_{t}^{q} u(t)=f\left(t, u(t),{ }^{c} D_{t}^{\gamma} u(t)\right), \quad t \in J^{\prime}, \\
\Delta u\left(t_{k}\right)=I_{k}\left(u\left(t_{k}\right),{ }^{c} D_{t}^{\gamma} u\left(t_{k}\right)\right), \\
\Delta^{c} D_{t}^{\gamma} u\left(t_{k}\right)=J_{k}\left(u\left(t_{k}\right),{ }^{c c} D_{t}^{\gamma} u\left(t_{k}\right)\right), \quad k=1,2, \ldots, m, \\
a u(0)-b u(1)=0, a D_{t}^{\gamma} u(0)-b D_{t}^{\gamma} u(1)=0,
\end{array}\right.
$$

where ${ }^{c} D_{t}^{q}$ and ${ }^{c} D_{t}^{\gamma}$ represent the common Caputo derivatives of orders $q$ and $\gamma$, and $1<q<2,0<\gamma<1$, and $J=$ $[0,1], 0=\quad t_{0}<t_{1}<t_{2}<\cdots<t_{m}<\quad t_{m+1}=1, J^{\prime}=J$ $\left\{t_{1}, t_{2}, \ldots, t_{m}\right\}$. Here, $f: J \times \mathbb{R} \times \mathbb{R} \longrightarrow \mathbb{R}$ and $I_{k}, J_{k}: \mathbb{R} \times \mathbb{R}$ $\longrightarrow \mathbb{R}$ are continuous functions. Now, $\Delta u\left(t_{k}\right)=u\left(t_{k}^{+}\right)-u$ $\left(t_{k}^{-}\right)$, where $u\left(t_{k}^{+}\right)$and $u\left(t_{k}^{-}\right)$denote the right limit and the left limit of $u(t)$ at the impulsive point $t_{k}$. Also, $\Delta^{c} D_{t}^{\gamma} u\left(t_{k}\right)={ }^{c} D_{t}^{\gamma} u\left(t_{k}^{+}\right)-{ }^{c} D_{t}^{\gamma} u\left(t_{k}^{-}\right)$, where $\quad{ }^{c} D_{t}^{\gamma} u\left(t_{k}^{+}\right)$ and ${ }^{c} D_{t}^{\gamma} u\left(t_{k}^{-}\right)$denote the right limit and the left limit of ${ }^{c} D_{t}^{\gamma} u(t)$ at the impulsive point $t_{k}$. If $u\left(t_{k}^{-}\right)$and ${ }^{c} D_{t}^{\gamma} u\left(t_{k}^{-}\right)$ exist, we let $u\left(t_{k}\right)=u\left(t_{k}^{-}\right)$and ${ }^{c} D_{t}^{\gamma} u\left(t_{k}\right)={ }^{c} D_{t}^{\gamma} u$ $\left(t_{k}^{-}\right)$, where $k=1,2, \ldots, m$. Also, $a$ and $b$ are two real constants with $b>a>0$.
The theory of fractional differential equation has received a lot of attention because of its wide application in mathematical models (see [1-27] and the references therein). Fractional-order impulsive differential equations are a natural generalization of the case of nonimpulses and are used to describe sudden changes in their states, such as in optimal control, population dynamics, biological systems, financial systems, and mechanical systems with impact. We refer the reader to [28-36] and the references therein. In particular, Bai et al. [37] investigated a mixed boundary value problem of nonlinear impulsive fractional differential equation:

$$
\left\{\begin{array}{l}
{ }^{c} D_{0^{+}}^{q} u(t)=f(t, u(t)), \quad t \in J^{\prime}, \\
\Delta u\left(t_{k}\right)=I_{k}\left(u\left(t_{k}\right)\right), \Delta u^{\prime}\left(t_{k}\right)=J_{k}\left(u\left(t_{k}\right)\right), \quad k=1,2, \ldots, m, \\
u(0)+u(1)=0, \\
u^{\prime}(0)+u^{\prime}(1)=0,
\end{array}\right.
$$

and some sufficient conditions on the existence and uniqueness of solutions for problem (2) are obtained under Lipschitz conditions. In [38], Zhang and Xu studied the following impulsive periodic boundary value problem with the Caputo fractional derivative: 


$$
\left\{\begin{array}{l}
{ }^{c} D_{t}^{q} u(t)=f(t, u(t)), \quad t \in J^{\prime}, \\
\Delta u\left(t_{k}\right)=I_{k}\left(u\left(t_{k}\right)\right), \Delta u^{\prime}\left(t_{k}\right)=J_{k}\left(u\left(t_{k}\right)\right), \quad k=1,2, \ldots, m, \\
a u(0)-b u(1)=0, \\
a u^{\prime}(0)-b u^{\prime}(1)=0,
\end{array}\right.
$$

using Green's function in [36], and via the symmetry property of Green's function and topological degree theory, the authors obtained the existence of positive solutions for (3) when the growth of $f$ is superlinear and sublinear.

Inspired by the above research studies, in this paper, we consider fractional-order impulsive differential equations with generalized periodic boundary value conditions (1), where the nonlinear term, impulse terms, and periodic boundary conditions all depend on unknown functions and the lower-order fractional derivative of unknown functions. This is obviously more general and more widely applied, but it is also more complex and difficult to solve. Compared with (1), the nonlinear term, pulse term, and periodic boundary conditions of (3) are all independent of fractional derivatives, so it is a special form of (1). In this paper, we first give an equivalent integral form of solutions for problem (1) using some new Green's functions. Next, we present some sufficient conditions for the existence of solutions for problem (1), where the nonlinear and impulse terms satisfy some nonlinear and linear growth conditions, which are different from the conditions in [36-38]. Finally, we present three examples to illustrate our main results.

\section{Preliminaries and Lemmas}

In this section, we only present some necessary definitions and lemmas about fractional calculus.

Definition 1 (see $[39,40])$. The Riemann-Liouville fractional integral of order $\alpha>0$ for a function $f:(0, \infty) \longrightarrow \mathbb{R}$ is defined as

$$
I_{0+}^{\alpha} f(t)=\frac{1}{\Gamma(\alpha)} \int_{0}^{t}(t-s)^{\alpha-1} f(s) \mathrm{d} s,
$$

where $\Gamma(\cdot)$ is the Euler gamma function.

Definition 2 (see $[39,40]$ ). The Caputo fractional derivative of order $\alpha>0$ for a continuous and $n$-order differentiable function $f:(0, \infty) \longrightarrow \mathbb{R}$ is defined as

$$
{ }^{c} D_{t}^{\alpha} f(t)=\frac{1}{\Gamma(n-\alpha)} \int_{0}^{t}(t-s)^{n-\alpha-1} f^{(n)}(s) \mathrm{d} s,
$$

where $\Gamma(\cdot)$ is the Euler gamma function and $n=[\alpha]+1$, where $[\alpha]$ is the smallest integer greater than or equal to $\alpha$.

Lemma 1 (see $[39,40])$. Let $\alpha>0$. The differential equation ${ }^{c} D_{t}^{\alpha} u(t)=0$ has a unique solution:

$$
u(t)=c_{0}+c_{1} t+\cdots+c_{n-1} t^{n-1}
$$

for some $c_{i} \in \mathbb{R}(i=0,1,2, \ldots, n-1)$, where $n=[\alpha]+1$.

Lemma 2 Let $y \in C(J)$ and $1<q<2$. The unique solution of the following periodic boundary value problem

$$
\left\{\begin{array}{l}
{ }^{c} D_{t}^{q} u(t)=y(t), \quad t \in J^{\prime}, \\
\Delta u\left(t_{k}\right)=I_{k}, \Delta^{c} D_{t}^{\gamma} u\left(t_{k}\right)=J_{k}, \quad k=1,2, \ldots, m, \\
a u(0)-b u(1)=0, a D_{t}^{\gamma} u(0)-b D_{t}^{\gamma} u(1)=0, \quad 0<a<b,
\end{array}\right.
$$

is expressed by

$$
u(t)=\int_{0}^{1} K_{1}(t, s) y(s) \mathrm{d} s+\sum_{i=1}^{m} K_{2}\left(t, t_{i}\right) J_{i}+\sum_{i=1}^{m} K_{3}\left(t, t_{i}\right) I_{i}, \quad t \in J,
$$

where

$$
\begin{aligned}
& K_{1}(t, s)= \begin{cases}\frac{(t-s)^{q-1}}{\Gamma(q)}-\frac{b(1-s)^{q-1}}{(b-a) \Gamma(q)}-\frac{\Gamma(2-\gamma)(1-s)^{q-\gamma-1}}{\Gamma(q-\gamma)}\left(t-\frac{b}{b-a}\right), & 0 \leq s \leq t \leq 1, \\
-\frac{b(1-s)^{q-1}}{(b-a) \Gamma(q)}-\frac{\Gamma(2-\gamma)(1-s)^{q-\gamma-1}}{\Gamma(q-\gamma)}\left(t-\frac{b}{b-a}\right), & 0 \leq t \leq s \leq 1,\end{cases} \\
& K_{2}\left(t, t_{i}\right)= \begin{cases}\frac{a \Gamma(2-\gamma) t_{i}}{(b-a) t_{i}^{1-\gamma},} & 0<t_{i}<t \leq 1, i=1,2, \ldots, m, \\
\frac{\Gamma(2-\gamma)}{t_{i}^{1-\gamma}}\left(\frac{b t_{i}}{b-a}-t\right), & 0 \leq t \leq t_{i}<1, i=1,2, \ldots, m,\end{cases} \\
& K_{3}\left(t, t_{i}\right)= \begin{cases}-\frac{a}{a-b}, & 0<t_{i}<t \leq 1, i=1,2, \ldots, m, \\
-\frac{b}{a-b}, & 0 \leq t \leq t_{i}<1, i=1,2, \ldots, m .\end{cases}
\end{aligned}
$$


Furthermore,

$$
{ }^{c} D_{t}^{\gamma} u(t)=\int_{0}^{t} H_{1}(t, s) y(s) \mathrm{d} s+\sum_{i=1}^{m} H_{2}\left(t, t_{i}\right) J_{i},
$$

where

$$
\begin{aligned}
& H_{1}(t, s)= \begin{cases}\frac{(t-s)^{q-\gamma-1}}{\Gamma(q-\gamma)}-\frac{(1-s)^{q-\gamma-1} t^{1-\gamma}}{\Gamma(q-\gamma)}, & 0 \leq s \leq t \leq 1, \\
-\frac{(1-s)^{q-\gamma-1} t^{1-\gamma}}{\Gamma(q-\gamma)}, & 0 \leq t \leq s \leq 1,\end{cases} \\
& H_{2}\left(t, t_{i}\right)= \begin{cases}0, & 0<t_{i}<t \leq 1, i=1,2, \ldots, m, \\
\left(\frac{t}{t_{i}}\right)^{1-\gamma}, & 0 \leq t \leq t_{i}<1, i=1,2, \ldots, m .\end{cases}
\end{aligned}
$$

$$
\begin{aligned}
a c_{0}-b c_{m} & =b d_{m}-b I_{0+}^{q} y(1), \\
d_{m} & =\Gamma(2-\gamma) I_{0+}^{q-\gamma} y(1) .
\end{aligned}
$$

Applying the right fractional-order impulsive condition of (7), we obtain

$$
\begin{aligned}
& d_{k-1}-d_{k}=\frac{\Gamma(2-\gamma)}{t_{k}^{1-\gamma}} J_{k}, \\
& c_{k-1}-c_{k}=I_{k}-\frac{\Gamma(2-\gamma)}{t_{k}^{1-\gamma}} J_{k} .
\end{aligned}
$$

From (15) and (16), after a recursive calculation, we have

$$
d_{0}=d_{m}+\Gamma(2-\gamma) \sum_{i=1}^{m} \frac{J_{i}}{t_{i}^{1-\gamma}}=\Gamma(2-\gamma) I_{0+}^{q-\gamma} y(1)+\Gamma(2-\gamma) \sum_{i=1}^{m} \frac{J_{i}}{t_{i}^{1-\gamma}} .
$$

Similar to (18), we see that

$$
d_{k}=d_{0}-\Gamma(2-\gamma) \sum_{i=1}^{k} \frac{J_{i}}{t_{i}^{1-\gamma}}=\Gamma(2-\gamma) I_{0+}^{q-\gamma} y(1)+\Gamma(2-\gamma) \sum_{i=k+1}^{m} \frac{J_{i}}{t_{i}^{1-\gamma}} \text {. }
$$

From (13), (14), and (16), we have

$$
c_{0}=\frac{b}{b-a}\left[I_{0+}^{q} y(1)-\Gamma(2-\gamma) I_{0+}^{q-\gamma} y(1)+\sum_{i=1}^{m}\left(I_{i}-\Gamma(2-\gamma) t_{i}^{\gamma} J_{i}\right)\right] \text {. }
$$

From (17) and (20), after a recursive calculation, we have

From (12) and (13), according to (7), we obtain

$$
\begin{aligned}
c_{k}=c_{0}-\sum_{i=1}^{k}\left[I_{i}-\Gamma(2-\gamma) t_{i}^{\gamma} J_{i}\right] & =\frac{b}{b-a}\left[I_{0+}^{q} y(1)-\Gamma(2-\gamma) I_{0+}^{q-\gamma} y(1)\right] \\
& +\frac{a}{b-a} \sum_{i=1}^{k}\left[I_{i}-\Gamma(2-\gamma) t_{i}^{\gamma} J_{i}\right]+\frac{b}{b-a} \sum_{i=1}^{m}\left[I_{i}-\Gamma(2-\gamma) t_{i}^{\gamma} J_{i}\right] .
\end{aligned}
$$

For $t \in J_{0}=\left[t_{0}, t_{1}\right]$, substituting (18) and (20) into (12) and (13), we obtain

$$
\begin{aligned}
u(t)= & I_{0+}^{q} y(t)-\frac{b}{b-a} I_{0+}^{q} y(1)-\Gamma(2-\gamma)\left(t-\frac{b}{b-a}\right) I_{0+}^{q-\gamma} y(1) \\
& -\sum_{i=1}^{m} \frac{\Gamma(2-\gamma)}{t_{i}^{1-\gamma}}\left(t-\frac{b t_{i}}{b-a}\right) J_{i}-\frac{b}{b-a} \sum_{i=1}^{m} I_{i} \\
& -\Gamma(2-\gamma)\left(t-\frac{b}{b-a}\right)\left(\int_{0}^{t}+\int_{t}^{1}\right) \frac{(1-s)^{q-\gamma-1}}{\Gamma(q-\gamma)} y(s) \mathrm{d} s+\sum_{i=1}^{m} \frac{\Gamma(2-\gamma)}{t_{i}^{1-\gamma}}\left(\frac{b t_{i}}{b-a}-t\right) J_{i}-\frac{b}{b-a} \sum_{i=1}^{m} I_{i}
\end{aligned}
$$




$$
\begin{aligned}
& =\int_{0}^{t} K_{1}(t, s) y(s) \mathrm{d} s+\sum_{i=1}^{m} K_{2}\left(t, t_{i}\right) J_{i}+\sum_{i=1}^{m} K_{3}\left(t, t_{i}\right) I_{i}, \\
{ }^{c} D_{t}^{\gamma} u(t) & =I_{0+}^{q-\gamma} y(t)-\left[I_{0+}^{q-\gamma} y(1)+\sum_{i=1}^{m} \frac{J_{i}}{t_{i}^{1-\gamma}}\right] t^{1-\gamma} \\
& =\int_{0}^{t} \frac{(t-s)^{q-\gamma-1}}{\Gamma(q-\gamma)} y(s) \mathrm{d} s-\left(\int_{0}^{t}+\int_{t}^{1}\right) \frac{(1-s)^{q-\gamma-1} t^{1-\gamma}}{\Gamma(q-\gamma)} y(s) \mathrm{d} s-\sum_{i=1}^{m}\left(\frac{t}{t_{i}}\right)^{1-\gamma} J_{i} \\
& =\int_{0}^{t} H_{1}(t, s) y(s) \mathrm{d} s+\sum_{i=1}^{m} H_{2}\left(t, t_{i}\right) J_{i},
\end{aligned}
$$

where $K_{1}(t, s), K_{2}\left(t, t_{i}\right), K_{3}\left(t, t_{i}\right), H_{1}(t, s)$, and $H_{2}\left(t, t_{i}\right)$ are defined by (7) and (9).
For $J_{k}=\left[t_{k}, t_{k+1}\right], k=1,2, \ldots, m$, substituting (20) and (18) into (11) and (12), we have

$$
\begin{aligned}
& u(t)=I_{0+}^{q} y(t)-\frac{b}{b-a} I_{0+}^{q} y(1)-\Gamma(2-\gamma)\left(t-\frac{b}{b-a}\right) I_{0+}^{q-\gamma} y(1) \\
& +\frac{a}{b-a} \sum_{i=1}^{k} \Gamma(2-\gamma) t_{k}^{\gamma} J_{i}-\sum_{i=1}^{m} \frac{\Gamma(2-\gamma)}{t_{i}^{1-\gamma}}\left(t-\frac{b t_{i}}{b-a}\right) J_{i}-\frac{a}{b-a} \sum_{i=1}^{k} I_{i}-\frac{b}{b-a} \sum_{i=1}^{m} I_{i} \\
& =\int_{0}^{t} \frac{(t-s)^{q-1}}{\Gamma(q)} y(s) \mathrm{d} s-\frac{b}{b-a}\left(\int_{0}^{t}+\int_{t}^{1}\right) \frac{(1-s)^{q-1}}{\Gamma(q)} y(s) \mathrm{d} s-\Gamma(2-\gamma)\left(t-\frac{b}{b-a}\right) \\
& \cdot\left(\int_{0}^{t}+\int_{t}^{1}\right) \frac{(1-s)^{q-\gamma-1}}{\Gamma(q-\gamma)} y(s) \mathrm{d} s+\frac{a}{b-a} \sum_{i=1}^{k} \frac{\Gamma(2-\gamma) t_{i}}{t_{i}^{1-\gamma}} J_{i}+\sum_{i=1}^{m} \frac{\Gamma(2-\gamma)}{t_{i}^{1-\gamma}}\left(\frac{b t_{i}}{b-a}-t\right) J_{i} \\
& -\frac{a}{b-a} \sum_{i=1}^{k} I_{i}-\frac{b}{b-a} \sum_{i=1}^{m} I_{i}=\int_{0}^{t} K_{1}(t, s) y(s) \mathrm{d} s+\sum_{i=1}^{m} K_{2}\left(t, t_{i}\right) J_{i}+\sum_{i=1}^{m} K_{3}\left(t, t_{i}\right) I_{i}, \\
& { }^{c} D_{t}^{\gamma} u(t)=I_{0+}^{q-\gamma} y(t)-\left[I_{0+}^{q-\gamma} y(1)+\sum_{i=1}^{m} \frac{J_{i}}{t_{i}^{1-\gamma}}\right] t^{1-\gamma} \\
& =\int_{0}^{t} \frac{(t-s)^{q-\gamma-1}}{\Gamma(q-\gamma)} y(s) \mathrm{d} s-\left(\int_{0}^{t}+\int_{t}^{1}\right) \frac{(1-s)^{q-\gamma-1} t^{1-\gamma}}{\Gamma(q-\gamma)} y(s) \mathrm{d} s-\sum_{i=1}^{m}\left(\frac{t}{t_{i}}\right)^{1-\gamma} J_{i} \\
& =\int_{0}^{1} H_{1}(t, s) y(s) \mathrm{d} s+\sum_{i=1}^{m} H_{2}\left(t, t_{i}\right) J_{i},
\end{aligned}
$$

where $K_{1}(t, s), K_{2}\left(t, t_{i}\right), K_{3}\left(t, t_{i}\right), H_{1}(t, s)$, and $H_{2}\left(t, t_{i}\right)$ are defined by (9) and (11). The proof is completed.

Lemma 3. Let $0<a<b<+\infty$. Then, $K_{1}(t, s)+K_{2}\left(t, t_{i}\right)$ and $K_{3}\left(t, t_{i}\right)$ and $H_{1}(t, s)$ and $H_{2}\left(t, t_{i}\right)$ defined as in (9) and (11) are continuous, and the following inequalities hold:

(i) $\left|K_{1}(t, s)\right| \leq\left((2 b-a)(1-s)^{q-1} /(b-a) \Gamma(q)\right)+((2 b$ $\left.-a) \Gamma(2-\gamma)(1-s)^{q-\gamma-1} /(b-a) \Gamma(q-\gamma)\right),\left|H_{1}(t, s)\right|$ $\leq\left(2(1-s)^{q-\gamma-1} / \Gamma(q-\gamma)\right), t, s \in J$ 
(ii) $\left|K_{2}\left(t, t_{i}\right)\right| \leq b \Gamma(2-\gamma) / b-a,\left|H_{2}\left(t, t_{i}\right)\right| \leq 1,\left|K_{3}\left(t, t_{i}\right)\right| \quad$ Proof. Directly observe that $\leq b / b-a, t, t_{i} \in J$

$$
\begin{aligned}
\left|K_{1}(t, s)\right| & \frac{(1-s)^{q-1}}{\Gamma(q)}+\frac{b(1-s)^{q-1}}{(b-a) \Gamma(q)}+\frac{\Gamma(2-\gamma)(1-s)^{q-\gamma-1}}{\Gamma(q-\gamma)}\left(1+\frac{b}{b-a}\right) \\
& \leq \frac{(2 b-a)(1-s)^{q-1}}{(b-a) \Gamma(q)}+\frac{(2 b-a) \Gamma(2-\gamma)(1-s)^{q-\gamma-1}}{(b-a) \Gamma(q-\gamma)}, \quad t, s \in J, \\
\left|H_{1}(t, s)\right| & \leq \frac{(1-s)^{q-\gamma-1}}{\Gamma(q-\gamma)}+\frac{(1-s)^{q-\gamma-1} t^{1-\gamma}}{\Gamma(q-\gamma)} \leq \frac{\left(1+t^{1-\gamma}\right)(1-s)^{q-\gamma-1}}{\Gamma(q-\gamma)}, \quad \leq \frac{2(1-s)^{q-\gamma-1}}{\Gamma(q-\gamma)}, t, s \in J, \\
\left|K_{2}\left(t, t_{i}\right)\right| & \leq \frac{\Gamma(2-\gamma)}{t_{i}^{1-\gamma}} \frac{b t_{i}}{b-a} \leq \frac{b \Gamma(2-\gamma)}{b-a} t_{i}^{\gamma} \leq \frac{b \Gamma(2-\gamma)}{b-a}, \quad t, t_{i} \in J, \\
\left|K_{3}\left(t, t_{i}\right)\right| & \leq \frac{b}{a-b}, \quad t, t_{i} \in J, \\
\left|H_{2}\left(t, t_{i}\right)\right| & \leq\left(\frac{t}{t_{i}}\right)^{1-\gamma} \leq 1, \quad t, t_{i} \in J .
\end{aligned}
$$

Let $\quad E=\left\{u: J \longrightarrow \mathbb{R} \mid u \in C\left(J^{\prime}\right), \quad{ }^{c} D_{t}^{\gamma} u(t) \in C\left(J^{\prime}\right)\right.$, and $u\left(t_{k}^{-}\right), u\left(t_{k}^{+}\right),{ }^{c} D_{t}^{\gamma} u\left(t_{k}^{-}\right)$, and ${ }^{c} D_{t}^{\gamma} u\left(t_{k}^{+}\right)$exist, where $k=1,2, \ldots, m\}$. Note [35] that $E$ is a Banach space equipped with the norm

$$
\|u\|=\sup _{t \in J}|u(t)|+\left.\sup _{t \in J}\right|^{c} D_{t}^{\gamma} u(t) \mid .
$$

Lemma 4. If the function $f\left(t, u,{ }^{c} D_{t}^{\gamma} u(t)\right)$ is continuous, then $u \in E$ is a solution of (1) if and only if $u \in E$ is a solution of the following integral equation:

$$
\begin{aligned}
u(t)= & \int_{0}^{1} K_{1}(t, s) f\left(s, u(s),{ }^{c} D_{t}^{\gamma} u(s)\right) d s+\sum_{i=1}^{m} K_{2}\left(t, t_{i}\right) J_{i}\left(u\left(t_{i}\right),{ }^{c} D_{t}^{\gamma} u\left(t_{i}\right)\right) \\
& +\sum_{i=1}^{m} K_{3}\left(t, t_{i}\right) I_{i}\left(u\left(t_{i}\right),{ }^{c} D_{t}^{\gamma} u\left(t_{i}\right)\right) .
\end{aligned}
$$

Proof. Assume that $u$ satisfies (1). From Lemma 2, we see that $u$ satisfies integral equation (26).

Conversely, assume that $u$ satisfies integral equation (26). Applying Definition 2, by a direct fractional derivative computation, it follows that the solution given by (26) and (2) satisfies (1).

Define an operator $T: E \longrightarrow E$ as

$$
\begin{aligned}
(T u)(t)= & \int_{0}^{1} K_{1}(t, s) f\left(s, u(s),{ }^{c} D_{t}^{\gamma} u(s)\right) \mathrm{d} s+\sum_{i=1}^{m} K_{2}\left(t, t_{i}\right) J_{i}\left(u\left(t_{i}\right),{ }^{c} D_{t}^{\gamma} u\left(t_{i}\right)\right) \\
& +\sum_{i=1}^{m} K_{3}\left(t, t_{i}\right) I_{i}\left(u\left(t_{i}\right),{ }^{c} D_{t}^{\gamma} u\left(t_{i}\right)\right), \\
\left({ }^{c} D_{t}^{\gamma} T u\right)(t)= & \int_{0}^{t} H_{1}(t, s) f\left(s, u(s),{ }^{c} D_{t}^{\gamma} u(s)\right) \mathrm{d} s+\sum_{i=1}^{m} H_{2}\left(t, t_{i}\right) J_{i}\left(u\left(t_{i}\right),{ }^{c} D_{t}^{\gamma} u\left(t_{i}\right)\right) .
\end{aligned}
$$


It is easy to prove that the function $u$ is a solution of (1) if and only if $u$ is a fixed point of the operator $T$.

For convenience, we list some hypotheses:

(B1) $0<a<b<+\infty, 1<q<2,0<\gamma<1$ with $q-\gamma>1$

(B2) $f: J \times \mathbb{R} \times \mathbb{R} \longrightarrow \mathbb{R}$ and $I_{k}, J_{k}: \mathbb{R} \times \mathbb{R} \longrightarrow \mathbb{R}$ are continuous functions

Lemma 5. Assume that (B1) and (B2) hold. Then, the operator $T: E \longrightarrow$ E defined as in (27) is completely continuous.
Proof. We divide the proof into three steps. Set $\Omega_{r}=\{u \in E,\|u\| \leq r\}$ for some $r>0$. The steps are as follows:

(i) Step 1. T is continuous from the continuity of the functions $K_{1}, K_{2}, K_{3}, H_{1}, H_{2}, f, I_{k}, J_{k}$.

(ii) Step 2. $T$ is uniformly bounded. Now, for $u \in \Omega_{r}$ we have $\left|f\left(t, u,{ }^{c} D_{t}^{\gamma} u\right)\right| \leq m_{1},\left|J_{k}\right| \leq m_{2},\left|I_{k}\right| \leq m_{3}$, where $m_{i}>0, i=1,2,3$.

In fact, for each $t \in J_{k}=\left[t_{k}, t_{k+1}\right], u \in \Omega_{r}$, $k=0,1,2, \ldots, m$, from Lemma 3 , we have

$$
\begin{aligned}
|(T u)(t)| \leq & \int_{0}^{1}\left|K_{1}(t, s) f\left(s, u(s),{ }^{c} D_{t}^{\gamma} u(s)\right)\right| \mathrm{d} s+\sum_{i=1}^{m}\left|K_{2}\left(t, t_{i}\right) J_{i}\left(u\left(t_{i}\right),{ }^{c} D_{t}^{\gamma} u\left(t_{i}\right)\right)\right| \\
& +\sum_{i=1}^{m}\left|K_{3}\left(t, t_{i}\right) I_{i}\left(u\left(t_{i}\right),{ }^{c} D_{t}^{\gamma} u\left(t_{i}\right)\right)\right| \leq m_{1} \int_{0}^{1}\left|K_{1}(t, s)\right| \mathrm{d} s+m_{2} \sum_{i=1}^{m}\left|K_{2}\left(t, t_{i}\right)\right|+m_{3} \sum_{i=1}^{m}\left|K_{3}\left(t, t_{i}\right)\right|, \\
\left|\left({ }^{c} D_{t}^{\gamma} T u\right)(t)\right| \leq & \int_{0}^{1}\left|H_{1}(t, s) f\left(s, u(s),{ }^{c} D_{t}^{\gamma} u(s)\right)\right| \mathrm{d} s+\sum_{i=1}^{m}\left|H_{2}\left(t, t_{i}\right) J_{i}\left(u\left(t_{i}\right),{ }^{c} D_{t}^{\gamma} u\left(t_{i}\right)\right)\right| \\
\leq & m_{1} \int_{0}^{1}\left|H_{1}(t, s)\right| \mathrm{d} s+m_{2} \sum_{i=1}^{m}\left|H_{2}\left(t, t_{i}\right)\right|
\end{aligned}
$$

which and Lemma 4 imply that

$$
\begin{aligned}
\|T u\| & =\sup _{t \in J}|(T u)(t)|+\sup _{t \in J}\left|\left({ }^{c} D_{t}^{\gamma} T u\right)(t)\right| \\
& \leq m_{1} \int_{0}^{1}\left|K_{1}(t, s)\right| \mathrm{d} s+m_{2} \sum_{i=1}^{m}\left|K_{2}\left(t, t_{i}\right)\right|+m_{3} \sum_{i=1}^{m}\left|K_{3}\left(t, t_{i}\right)\right|+m_{1} \int_{0}^{1}\left|H_{1}(t, s)\right| \mathrm{d}+m_{2} \sum_{i=1}^{m}\left|H_{2}\left(t, t_{i}\right)\right| \\
\leq & m_{1} \int_{0}^{1}\left[\left|K_{1}(t, s)\right|+\left|H_{1}(t, s)\right|\right] \mathrm{d}+m_{2} \sum_{i=1}^{m}\left[\left|K_{2}\left(t, t_{i}\right)\right|+\left|H_{2}\left(t, t_{i}\right)\right|\right]+m_{3} \sum_{i=1}^{m}\left|K_{3}\left(t, t_{i}\right)\right| \\
\leq & m_{1} \int_{0}^{1}\left[\frac{(2 b-a)(1-s)^{q-1}}{(b-a) \Gamma(q)}+\frac{(2 b-a) \Gamma(2-\gamma)(1-s)^{q-\gamma-1}}{(b-a) \Gamma(q-\gamma)}+\frac{2(1-s)^{q-\gamma-1}}{\Gamma(q-\gamma)}\right] \mathrm{d} s \\
& +\frac{[b \Gamma(2-\gamma)+b-a] m m_{2}}{b-a}+\frac{b m m_{3}}{b-a}=m_{1}\left[\frac{(2 b-a)}{(b-a) \Gamma(q+1)}+\frac{(2 b-a) \Gamma(2-\gamma)}{(b-a) \Gamma(q-\gamma+1)}+\frac{2}{\Gamma(q-\gamma+1)}\right] \\
& +\frac{[b \Gamma(2-\gamma)+b-a] m m_{2}}{b-a}+\frac{b m m_{3}}{b-a}=M .
\end{aligned}
$$


(iii) Step 3. $T$ is equicontinuous. For any $t_{1}, t_{2} \in J_{k}, k=0,1, \ldots, m$, fixed $s \in J$ and for any $\epsilon>0$, there exists a constant $\delta>0$ such that for $\left|t_{1}-t_{2}\right|<\delta$, we have

$$
\begin{gathered}
\left|K_{1}\left(t_{1}, s\right)-K_{1}\left(t_{2}, s\right)\right|<\frac{\epsilon}{6 m_{1}}, \\
\left|K_{2}\left(t_{1}, t_{i}\right)-K_{2}\left(t_{2}, t_{i}\right)\right|<\frac{\epsilon}{6 m_{2} m}, \\
\left|K_{3}\left(t_{1}, t_{i}\right)-K_{3}\left(t_{2}, t_{i}\right)\right|<\frac{\epsilon}{6 m_{3} m}, \\
\left|H_{1}\left(t_{1}, s\right)-H_{1}\left(t_{2}, s\right)\right|<\frac{\epsilon}{4 m_{1}}, \\
\left|H_{2}\left(t_{1}, t_{i}\right)-H_{2}\left(t_{2}, t_{i}\right)\right|<\frac{\epsilon}{4 m_{2} m} .
\end{gathered}
$$

Then,

$$
\begin{aligned}
(T u)\left(t_{1}\right)-(T u)\left(t_{2}\right)= & \mid \int_{0}^{1}\left(K_{1}\left(t_{1}, s\right)-K_{1}\left(t_{2}, s\right)\right) f\left(s, u(s),{ }^{c} D_{t}^{\gamma} u(s)\right) \mathrm{d} s \\
& +\sum_{i=1}^{m}\left(K_{2}\left(t_{1}, t_{i}\right)-K_{2}\left(t_{2}, t_{i}\right)\right) J_{i}\left(u\left(t_{i}\right),{ }^{c} D_{t}^{\gamma} u\left(t_{i}\right)\right) \\
& +\sum_{i=1}^{m}\left(K_{3}\left(t_{1}, t_{i}\right)-K_{3}\left(t_{2}, t_{i}\right)\right) I_{i}\left(u\left(t_{i}\right),{ }^{c} D_{t}^{\gamma} u\left(t_{i}\right)\right) \mid \\
\leq & m_{1} \int_{0}^{1}\left|K_{1}\left(t_{1}, s\right)-K_{1}\left(t_{2}, s\right)\right| \mathrm{d} s+m_{2} m\left|K_{2}\left(t_{1}, t_{i}\right)-K_{2}\left(t_{2}, t_{i}\right)\right| \\
& +m_{3} m\left|K_{3}\left(t_{1}, t_{i}\right)-K_{3}\left(t_{2}, t_{i}\right)\right|<\frac{\epsilon}{6}+\frac{\epsilon}{6}+\frac{\epsilon}{6}=\frac{\epsilon}{2}, \\
\left|\left({ }^{c} D_{t}^{\gamma} T u\right)\left(t_{1}\right)-\left({ }^{c} D_{t}^{\gamma} T u\right)\left(t_{2}\right)\right|= & \mid \int_{0}^{1}\left(H_{1}\left(t_{1}, s\right)-H_{1}\left(t_{2}, s\right)\right) f\left(s, u(s),{ }^{c} D_{t}^{\gamma} u(s)\right) \mathrm{d} s \\
& +\sum_{i=1}^{m}\left(H_{2}\left(t_{1}, t_{i}\right)-H_{2}\left(t_{2}, t_{i}\right)\right) J_{i}\left(u\left(t_{i}\right),{ }^{c} D_{t}^{\gamma} u\left(t_{i}\right)\right) \mid \\
\leq & m_{1} \int_{0}^{1}\left|H_{1}\left(t_{1}, s\right)-H_{1}\left(t_{2}, s\right)\right| \mathrm{d} s+m_{2} m\left|H_{2}\left(t_{1}, t_{i}\right)-H_{2}\left(t_{2}, t_{i}\right)\right|<\frac{\epsilon}{4}+\frac{\epsilon}{4}=\frac{\epsilon}{2} .
\end{aligned}
$$

Thus,

$$
\left\|(T u)\left(t_{1}\right)-(T u)\left(t_{2}\right)\right\|<\varepsilon .
$$

which implies that $T\left(\Omega_{r}\right)$ is equicontinuous on any subinterval $J_{k}, k=0,1, \ldots, m$.

From the Arzela-Ascoli theorem, we deduce that $T: E \longrightarrow E$ is completely continuous.
Lemma 6 (Schauder fixed-point theorem, see [41, 42]). Let $X$ be a real Banach space, $C \subset X$ be a nonempty closed bounded and convex subset, and $F: C \longrightarrow C$ be compact. Then, $T$ has at least one fixed point in $C$.

Lemma 7 (Krasnoselskii fixed point theorem, see [41, 42]). Let $\Omega$ be a closed convex and nonempty subset of a Banach space $X$. Let $\Phi$ and $\Psi$ be the operators such that (i) $\Phi x+\Psi y \in \Omega$ 
whenever $x, y \in \Omega$; (ii) $\Phi: \Omega \longrightarrow X$ is compact and continuous; and (iii) $\Psi$ is a contraction mapping. Then, there exists an $z \in \Omega$ such that $z=\Phi z+\Psi z$.

Lemma 8 (Banach's fixed point theorem, see [43]). Let $E$ be a Banach space, $\Omega \subset E$ be closed, and $F: \Omega \longrightarrow \Omega$ be a strict contraction, i.e., $|F x-F y| \leq k|x-y|$ for some $k \in(0,1)$ and all $x, y \in \Omega$. Then, $F$ has a unique fixed point in $\Omega$.

\section{Existence of the Solutions}

For convenience, we give the following symbols:

$$
\begin{aligned}
A_{i} & =\int_{0}^{1}\left[\frac{(2 b-a)(1-s)^{q-1}}{(b-a) \Gamma(q)}+\frac{(2 b-a) \Gamma(2-\gamma)(1-s)^{q-\gamma-1}}{(b-a) \Gamma(q-\gamma)}+\frac{2(1-s)^{q-\gamma-1}}{\Gamma(q-\gamma)}\right] a_{i}(s) \mathrm{d} s, \\
B_{i} & =\frac{[b \Gamma(2-\gamma)+b-a] m b_{i}}{b-a}, \\
C_{i} & =\frac{m b c_{i}}{b-a}, \quad i=0,1,2 .
\end{aligned}
$$

Now, we present our main theorems.

Theorem 1 Assume that (B1) and (B2) hold, and the following hypotheses are satisfied:
(C1) There exist three nonnegative functions $a_{0}, a_{1}, a_{2} \in L(J)$ and two constants $\lambda_{1}, \lambda_{2} \in(0,1)$ such that

$$
|f(t, u, v)| \leq a_{0}(t)+a_{1}(t)|u|^{\lambda_{1}}+a_{2}(t)|v|^{\lambda_{2}}, \quad \forall t \in J, u, v \in \mathbb{R}
$$

(C2) There exist eight positive constants $b_{1}, b_{2}, c_{1}, c_{2} \geq 0$

and $\mu_{1}, \mu_{2}, \nu_{1}, v_{2} \in(0,1)$ such that

$$
\begin{aligned}
& \left|I_{i}(u, v)\right| \leq b_{0}+b_{1}|u|^{\mu_{1}}+b_{2}|v|^{\mu_{2}}, \\
& \left|J_{i}(u, v)\right| \leq c_{0}+c_{1}|u|^{\nu_{1}}+c_{2}|v|^{\nu_{2}}, \quad i=1,2, \ldots, m, \forall u, v \in \mathbb{R} .
\end{aligned}
$$

Then, (1) has at least one solution in $E$.

Proof. Let

$$
\begin{aligned}
R_{1} & \geq \max \left\{7\left(A_{0}+B_{0}+C_{0}\right),\left(7 A_{1}\right)^{1 / 1-\lambda_{1}},\left(7 A_{2}\right)^{1 / 1-\lambda_{2}},\left(7 B_{1}\right)^{1 / 1-\mu_{1}},\left(7 B_{2}\right)^{1 / 1-\mu_{2}},\left(7 C_{1}\right)^{1 / 1-\nu_{1}},\left(7 C_{1}\right)^{1 / 1-\nu_{2}}\right\}, \\
\Omega_{R_{1}} & =\left\{u \in E:\|u\| \leq R_{1}\right\} .
\end{aligned}
$$

Now, $\Omega_{R_{1}}$ is a closed bounded convex subset of $E$.

For each $u \in \Omega_{R_{1}}$, from (C1) and (C2), we have

$$
\begin{aligned}
|(T u)(t)| \leq & \int_{0}^{1}\left|K_{1}(t, s) f\left(s, u(s),{ }^{c} D_{t}^{\gamma} u(s)\right)\right| \mathrm{d} s+\sum_{i=1}^{m}\left|K_{2}\left(t, t_{i}\right) J_{i}\left(u\left(t_{i}\right),{ }^{c} D_{t}^{\gamma} u\left(t_{i}\right)\right)\right| \\
& +\sum_{i=1}^{m}\left|K_{3}\left(t, t_{i}\right) I_{i}\left(u\left(t_{i}\right),{ }^{c} D_{t}^{\gamma} u\left(t_{i}\right)\right)\right| \leq \int_{0}^{1}\left|K_{1}(t, s)\right|\left[a_{0}(s)+a_{1}(s)|u(s)|^{\lambda_{1}}+\left.\left.a_{2}(s)\right|^{c} D_{t}^{\gamma} u(s)\right|^{\lambda_{2}}\right] \mathrm{d} s
\end{aligned}
$$




$$
\begin{aligned}
& +\sum_{i=1}^{m}\left|K_{2}\left(t, t_{i}\right)\right|\left[b_{0}+b_{1}|u(s)|^{\mu_{1}}+\left.\left.b_{2}\right|^{c} D_{t}^{\gamma} u(s)\right|^{\mu_{2}}\right]+\sum_{i=1}^{m}\left|K_{3}\left(t, t_{i}\right)\right|\left[c_{0}+c_{1}|u(s)|^{\nu_{1}}+\left.\left.c_{2}\right|^{c} D_{t}^{\gamma} u(s)\right|^{\nu_{2}}\right], \\
\left|\left({ }^{c} D_{t}^{\gamma} T u\right)(t)\right| \leq & \int_{0}^{1}\left|H_{1}(t, s) f\left(s, u(s),{ }^{c} D_{t}^{\gamma} u(s)\right)\right| \mathrm{d} s+\sum_{i=1}^{m}\left|H_{2}\left(t, t_{i}\right) J_{i}\left(u\left(t_{i}\right),{ }^{c} D_{t}^{\gamma} u\left(t_{i}\right)\right)\right| \\
\leq & \int_{0}^{1}\left|H_{1}(t, s)\right|\left[a_{0}(s)+a_{1}(s)|u(s)|^{\lambda_{1}}+a_{2}(s)\left|{ }^{c} D_{t}^{\gamma} u(s)\right|^{\lambda_{2}}\right] \mathrm{d} s \\
& +\sum_{i=1}^{m}\left|H_{2}\left(t, t_{i}\right)\right|\left[b_{0}+b_{1}|u(s)|^{\mu_{1}}+\left.\left.b_{2}\right|^{c} D_{t}^{\gamma} u(s)\right|^{\mu_{2}}\right] .
\end{aligned}
$$

From Lemma 3, we obtain that

$$
\begin{aligned}
\|T u\|= & \sup _{t \in J}|u(t)|+\sup _{t \in J}\left|\left({ }^{c} D_{t}^{\gamma} T u\right)(t)\right| \\
\leq & \int_{0}^{1}\left(\left|K_{1}(t, s)\right|+\left|H_{1}(t, s)\right|\right)\left(a_{0}(s)+a_{1}(s)\|u\|^{\lambda_{1}}+a_{2}(s)\|u\|^{\lambda_{2}}\right) \mathrm{d} s \\
& +\sum_{i=1}^{m}\left(\left|K_{2}\left(t, t_{i}\right)\right|+\left|H_{2}\left(t, t_{i}\right)\right|\right)\left(b_{0}+b_{1}\|u\|^{\mu_{1}}+b_{2}\|u\|^{\mu_{2}}\right) \\
& +\sum_{i=1}^{m}\left|K_{3}\left(t, t_{i}\right)\right|\left(c_{0}+c_{1}\|u\|^{\nu_{1}}+c_{2}\|u\|^{\nu_{2}}\right) \\
\leq & A_{0}+A_{1}\left\|R_{1}\right\|^{\lambda_{1}}+A_{2}\left\|R_{1}\right\|^{\lambda_{2}}+B_{0}+B_{1}\left\|R_{1}\right\|^{\mu_{1}}+B_{2}\left\|R_{1}\right\|^{\mu_{2}} \\
& +C_{0}+C_{1}\left\|R_{1}\right\|^{\nu_{1}}+C_{2}\left\|R_{1}\right\|^{\nu_{2}} \leq R_{1},
\end{aligned}
$$

which implies that $T\left(\Omega_{R_{1}}\right) \subset \Omega_{R_{1}}$.

From Lemmas 5 and $6, T$ has at least one fixed point in $\Omega_{R_{1}}$, so (1) has at least one solution in $E$.

$$
|f(t, u, v)| \leq a_{0}(t), \quad \forall t \in J, u, v \in \mathbb{R} .
$$

Theorem 2. Assume that (B1) and (B2) hold, and the following hypotheses are satisfied:

(C3) There exists a nonnegative function $a_{0} \in L(J)$, such that
(C4) There exist four positive constants $b_{1}, b_{2}, c_{1}, c_{2} \geq 0$ such that

$$
\begin{array}{ll}
\left|I_{i}\left(u_{1}, v_{1}\right)-I_{i}\left(u_{2}, v_{2}\right)\right| \leq b_{1}\left|u_{1}-u_{2}\right|+b_{2}\left|v_{1}-v_{2}\right|, & \forall u_{1}, u_{2}, v_{1}, v_{2} \in \mathbb{R}, i=1,2, \ldots, m, \\
\left|J_{i}\left(u_{1}, v_{1}\right)-J_{i}\left(u_{2}, v_{2}\right)\right| \leq c_{1}\left|u_{1}-u_{2}\right|+c_{2}\left|v_{1}-v_{2}\right|, & \forall u_{1}, u_{2}, v_{1}, v_{2} \in \mathbb{R}, i=1,2, \ldots, m .
\end{array}
$$

If $\Lambda=\sum_{i=1}^{2}\left(B_{i}+C_{i}\right)<1 / 2$, then (1) has at least one solution in $E$.
Proof. We first define the operators. For $u \in E$, let 


$$
\begin{aligned}
& (\Phi u)(t)=\int_{0}^{1} K_{1}(t, s) f\left(s, u(s),{ }^{c} D_{t}^{\gamma} u(s)\right) \mathrm{d} s \\
& \left(\Phi_{1} u\right)(t)=\int_{0}^{1} H_{1}(t, s) f\left(s, u(s),{ }^{c} D_{t}^{\gamma} u(s)\right) \mathrm{d} s \\
& (\Psi u)(t)=\sum_{i=1}^{m} K_{2}\left(t, t_{i}\right) J_{i}\left(u\left(t_{i}\right),{ }^{c} D_{t}^{\gamma} u\left(t_{i}\right)\right)+\sum_{i=1}^{m} K_{3}\left(t, t_{i}\right) I_{i}\left(u\left(t_{i}\right),{ }^{c} D_{t}^{\gamma} u\left(t_{i}\right)\right), \\
& \left(\Psi_{1} u\right)(t)=\sum_{i=1}^{m} H_{2}\left(t, t_{i}\right) J_{i}\left(u\left(t_{i}\right),{ }^{c} D_{t}^{\gamma} u\left(t_{i}\right)\right) .
\end{aligned}
$$

Now,

$$
\begin{aligned}
(T u)(t) & =(\Phi u)(t)+(\Psi u)(t), \\
\left({ }^{c} D_{t}^{\gamma} T u\right)(t) & ={ }^{c} D_{t}^{\gamma}(\Phi u)(t)+{ }^{c} D_{t}^{\gamma}(\Psi u)(t)=\left(\Phi_{1} u\right)(t)+\left(\Psi{ }_{1} u\right)(t) .
\end{aligned}
$$

Let

$$
\begin{aligned}
& M_{1}=\max _{1 \leq i \leq m}\left|I_{i}(0,0)\right|, \\
& M_{2}=\max _{1 \leq i \leq m}\left|J_{i}(0,0)\right| .
\end{aligned}
$$

Let

$$
\begin{gathered}
R_{2} \geq \max \left\{2 A_{0}, \frac{2 \Theta}{1-2 \Lambda}\right\}, \\
\Omega_{R_{2}}=\left\{u \in E:\|u\| \leq R_{2}\right\} .
\end{gathered}
$$

Note that $\Omega_{R_{2}}$ is a nonempty bounded closed convex subset of $E$.

From Lemma 5, $\Phi$ is completely continuous (i.e., condition (ii) of Lemma 7 is satisfied).

For any $u, v \in \Omega_{R_{2}}$, from hypothesis (C4), we have

$$
\begin{aligned}
|(\Psi u)(t)-(\Psi v)(t)| \leq & \sum_{i=1}^{m}\left|K_{2} \|\left(t, t_{i}\right) J_{i}\left(u\left(t_{i}\right),{ }^{c} D_{t}^{\gamma} u\left(t_{i}\right)\right)-J_{i}\left(v\left(t_{i}\right),{ }^{c} D_{t}^{\gamma} v\left(t_{i}\right)\right)\right| \\
& +\sum_{i=1}^{m}\left|K_{3} \|\left(t, t_{i}\right) I_{i}\left(u\left(t_{i}\right),{ }^{c} D_{t}^{\gamma} u\left(t_{i}\right)\right)-I_{i}\left(v\left(t_{i}\right),{ }^{c} D_{t}^{\gamma} v\left(t_{i}\right)\right)\right| \\
\leq & \sum_{i=1}^{m}\left|K_{2}\left(t, t_{i}\right)\right|\left(c_{1}|u(s)-v(s)|+c_{2}\left|{ }^{c} D_{t}^{\gamma} u(s)-{ }^{c} D_{t}^{\gamma} v(s)\right|\right) \\
& \cdot \sum_{i=1}^{m}\left|K_{3}\left(t, t_{i}\right)\right|\left(b_{1}|u(s)-v(s)|+b_{2}\left|{ }^{c} D_{t}^{\gamma} u(s)-{ }^{c} D_{t}^{\gamma} v(s)\right|\right) \\
\leq & \sum_{i=1}^{m}\left|K_{2}\left(t, t_{i}\right)\right|\left(c_{1}+c_{2}\right)\|u-v\|+\sum_{i=1}^{m}\left|K_{3}\left(t, t_{i}\right)\right|\left(b_{1}+b_{2}\right)\|u-v\| \\
\left|\left(\Psi_{1} u\right)(t)-\left(\Psi_{1} v\right)(t)\right| \leq & \sum_{i=1}^{m}\left|H_{2} \|\left(t, t_{i}\right) J_{i}\left(u\left(t_{i}\right),{ }^{c} D_{t}^{\gamma} u\left(t_{i}\right)\right)-J_{i}\left(v\left(t_{i}\right),{ }^{c} D_{t}^{\gamma} v\left(t_{i}\right)\right)\right| \\
\leq & \sum_{i=1}^{m}\left|H_{2}\left(t, t_{i}\right)\right|\left(c_{1}|u(s)-v(s)|+c_{2}\left|{ }^{c} D_{t}^{\gamma} u(s)-{ }^{c} D_{t}^{\gamma} v(s)\right|\right) \\
\leq & \sum_{i=1}^{m}\left|H_{2}\left(t, t_{i}\right)\right|\left(c_{1}+c_{2}\right)\|u-v\| .
\end{aligned}
$$


Therefore,

$$
\begin{aligned}
\|\Psi u-\Psi v\|= & \max _{t \in J}|\Psi u-\Psi v|+\max _{t \in J}\left|\Psi_{1} u-\Psi_{1} v\right| \\
\leq & \sum_{i=1}^{m}\left[\left|K_{2}\left(t, t_{i}\right)\right|+\left|H_{2}\left(t, t_{i}\right)\right|\right]\left(c_{1}+c_{2}\right)\|u-v\| \\
& +\sum_{i=1}^{m}\left|K_{3}\left(t, t_{i}\right)\right|\left(b_{1}+b_{2}\right)\|u-v\| \\
\leq & \left(C_{1}+C_{2}+B_{1}+B_{2}\right)\|u-v\|=\Lambda\|u-v\|,
\end{aligned}
$$

and since $\Lambda<1 / 2, \Psi$ is a contraction (so condition (iii) of Lemma 7 is satisfied).

For each $u \in \Omega_{R_{2}}$, from hypothesis (C3), we have

$$
\begin{aligned}
|(\Phi u)(t)| & \leq \int_{0}^{1}\left|K_{1}(t, s) f\left(s, u(s),{ }^{c} D_{t}^{\gamma} u(s)\right)\right| \mathrm{d} s \\
& \leq \int_{0}^{1}\left|K_{1}(t, s)\right| a_{0}(s) \mathrm{d} s, \\
\left|\left(\Phi_{1} u\right)(t)\right| & \leq \int_{0}^{1}\left|H_{1}(t, s) f\left(s, u(s),{ }^{c} D_{t}^{\gamma} u(s)\right)\right| \mathrm{d} s \\
& \leq \int_{0}^{1}\left|H_{1}(t, s)\right| a_{0}(s) \mathrm{d} s .
\end{aligned}
$$

Consequently,

$$
\|\Phi u\| \leq \int_{0}^{1}\left(\left|H_{1}(t, s)\right|+\left|H_{1}(t, s)\right|\right) a_{0}(s) \mathrm{d} s \leq A_{0} \leq \frac{R_{2}}{2} .
$$

For each $v \in \Omega_{R_{2}}$, we have

$$
\begin{aligned}
\|(\Psi v)\| \leq & \|(\Psi 0)-(\Psi v)\|+\|(\Psi 0)\| \\
\leq & \Lambda\|0-v\|+\sum_{i=1}^{m}\left(\left|K_{2}\left(t, t_{i}\right)\right|+\left|H_{2}\left(t, t_{i}\right)\right|\right) \max _{t \in J}\left|J_{i}(0,0)\right| \\
& +\sum_{i=1}^{m}\left|K_{3}\left(t, t_{i}\right)\right| \max _{t \in J}\left|I_{i}(0,0)\right| \leq \Lambda\|v\|+\Theta,
\end{aligned}
$$

where

$$
\Theta=m\left(\frac{b \Gamma(2-\gamma)+b-a}{b-a} M_{2}+\frac{b}{b-a} M_{1}\right) .
$$

Thus, for any $u, v \in \Omega_{R_{2}}$, we obtain

$$
\|(\Phi u)+(\Psi v)\| \leq\|(\Phi u)\|+\|(\Psi v)\| \leq A_{0}+\Lambda\|v\|+\Theta \leq R_{2},
$$

which implies that $\Phi u+\Psi v \in \Omega_{R_{2}}$ (so condition (i) of Lemma 7 is satisfied).

In view of Lemma 7 , there exists a $u \in \Omega_{R_{2}}$ such that $\Phi u+\Psi u=u$, so (1) has at least one solution in $E$.

Theorem 3. Assume that (B1), (B2), and (C4) hold and the following hypothesis is satisfied:

(C5) There exist two nonnegative functions $a_{1}, a_{2} \in L(J)$ such that

$$
\begin{array}{ll}
\left|f\left(t, u_{1}, v_{1}\right)-f\left(t, u_{2}, v_{2}\right)\right| \leq a_{1}(t)\left|u_{1}-u_{2}\right|+a_{2}(t)\left|v_{1}-v_{2}\right|, & \forall t \in J, u_{1}, u_{2}, v_{1}, v_{2} \in \mathbb{R} . \\
\text { If } \Pi=\left(A_{1}+A_{2}\right)+[b \Gamma(2-\gamma)+b-a]\left(c_{1}+c_{2}\right)+ & R_{3} \geq \frac{1}{1-\Pi}\left\{A^{\prime} M_{0}+B^{\prime}\right\}, \\
b\left(b_{1}+b_{2}\right) / b-a<1, \text { then (1) has a unique solution in } E . &
\end{array}
$$

where

Proof. Choose

$$
\begin{aligned}
& M_{0}=\max _{t \in J}|f(t, 0,0)|, \\
& M_{1}=\max _{1 \leq i \leq m}\left|I_{i}(0,0)\right|, \\
& M_{2}=\max _{1 \leq i \leq m}\left|J_{i}(0,0)\right|, \\
& A^{\prime}=\frac{(2 b-a)}{(b-a) \Gamma(q+1)}+\frac{(2 b-a) \Gamma(2-\gamma)}{(b-a) \Gamma(q-\gamma+1)}+\frac{2}{\Gamma(q-\gamma+1)}, \\
& B^{\prime}=\frac{b \Gamma(2-\gamma) M_{2}+b M_{1}+b-a}{b-a} .
\end{aligned}
$$


First, we show that $T \Omega_{R_{3}} \subset \Omega_{R_{3}}$, where $\Omega_{R_{3}}=\left\{u \in E,\|u\| \leq R_{3}\right\}$. For $u \in \Omega_{R_{3}}$, from hypotheses (C4) and (C5), we obtain

$$
\begin{aligned}
|(T u)(t)| \leq & \int_{0}^{1}\left|K_{1}(t, s)\right|\left[\left|f\left(s, u(s),{ }^{c} D_{t}^{\gamma} u(s)\right)-f(t, 0,0)\right|+|f(t, 0,0)|\right] \mathrm{d} s \\
& +\sum_{i=1}^{m}\left|K_{2}\left(t, t_{i}\right)\right|\left[\left|J_{i}\left(u\left(t_{i}\right),{ }^{c} D_{t}^{\gamma} u\left(t_{i}\right)\right)-J_{i}(0,0)\right|+\left|J_{i}(0,0)\right|\right] \\
& +\sum_{i=1}^{m}\left|K_{3}\left(t, t_{i}\right)\right|\left[\left|I_{i}\left(u\left(t_{i}\right),{ }^{c} D_{t}^{\gamma} u\left(t_{i}\right)\right)-I_{i}(0,0)\right|+\left|I_{i}(0,0)\right|\right] \\
\leq & \int_{0}^{1}\left|K_{1}(t, s)\right|\left[\left(a_{1}(t)+a_{2}(t)\right)\|u\|+M_{0}\right] \mathrm{d} s+\sum_{i=1}^{m}\left|K_{2}\left(t, t_{i}\right)\right|\left[\left(c_{1}+c_{2}\right)\|u\|+M_{2}\right] \\
& +\sum_{i=1}^{m} \| K_{3}\left(t, t_{i}\right)\left[\left(b_{1}+b_{2}\right)\|u\|+M_{1}\right], \\
\left|\left({ }^{c} D_{t}^{\gamma} T u\right)(t)\right| \leq & \int_{0}^{1}\left|H_{1}(t, s)\right|\left[\left|f\left(s, u(s),{ }^{c} D_{t}^{\gamma} u(s)\right)-f(t, 0,0)\right|+|f(t, 0,0)|\right] \mathrm{d} s \\
& +\sum_{i=1}^{m}\left|H_{2}\left(t, t_{i}\right)\right|\left[\left|J_{i}\left(u\left(t_{i}\right),{ }^{c} D_{t}^{\gamma} u\left(t_{i}\right)\right)-J_{i}(0,0)\right|+\left|J_{i}(0,0)\right|\right] \\
\leq & \int_{0}^{1}\left|H_{1}(t, s)\right|\left[\left(a_{1}(t)+a_{2}(t)\right)\|u\|+M_{0}\right] \mathrm{d} s \\
& +\sum_{i=1}^{m}\left|H_{2}\left(t, t_{i}\right)\right|\left[\left(c_{1}+c_{2}\right)\|u\|+M_{2}\right] .
\end{aligned}
$$

Then,

$$
\begin{aligned}
\|T u\| \leq & \int_{0}^{1}\left[\left|K_{1}(t, s)\right|+\left|H_{1}(t, s)\right|\right]\left[\left(a_{1}(t)+a_{2}(t)\right)\|u\|+M_{0}\right] \mathrm{d} s \\
& +\sum_{i=1}^{m}\left[\left|K_{2}\left(t, t_{i}\right)\right|+\left|H_{2}\left(t, t_{i}\right)\right|\right]\left[\left(c_{1}+c_{2}\right)\|u\|+M_{2}\right] \\
& +\sum_{i=1}^{m}\left|K_{3}\left(t, t_{i}\right)\right|\left[\left(b_{1}+b_{2}\right)\|u\|+M_{1}\right] \leq \Pi\|u\|+A^{\prime} M_{0}+B^{\prime} \leq R_{3},
\end{aligned}
$$

so $T \Omega_{R_{3}} \subset \Omega_{R_{3}}$.

Furthermore, from hypotheses (C4) and (C5), for all $u, v \in \Omega_{R_{3}}$, we have

$$
\begin{aligned}
|(T u)(t)-(T v)(t)| \leq & \int_{0}^{1}\left|K_{1}(t, s)\right|\left|f\left(s, u(s),{ }^{c} D_{t}^{\gamma} u(s)\right)-f\left(s, v(s),{ }^{c} D_{t}^{\gamma} v(s)\right)\right| \mathrm{d} s \\
& +\sum_{i=1}^{m}\left|K_{2}\left(t, t_{i}\right)\right|\left|J_{i}\left(u\left(t_{i}\right),{ }^{c} D_{t}^{\gamma} u\left(t_{i}\right)\right)-J_{i}\left(v\left(t_{i}\right),{ }^{c} D_{t}^{\gamma} v\left(t_{i}\right)\right)\right| \\
& +\sum_{i=1}^{m}\left|K_{3}\left(t, t_{i}\right)\right|\left|I_{i}\left(u\left(t_{i}\right),{ }^{c} D_{t}^{\gamma} u\left(t_{i}\right)\right)-I_{i}\left(v\left(t_{i}\right),{ }^{c} D_{t}^{\gamma} v\left(t_{i}\right)\right)\right|
\end{aligned}
$$




$$
\begin{aligned}
\leq & \int_{0}^{1}\left|K_{1}(t, s)\right|\left(a_{1}(t)+a_{2}(t)\right)\|u-v\| \mathrm{d} s+\sum_{i=1}^{m}\left|K_{2}\left(t, t_{i}\right)\right|\left(c_{1}+c_{2}\right)\|u-v\| \\
& +\sum_{i=1}^{m}\left|K_{3}\left\|\left(t, t_{i}\right)\left(b_{1}+b_{2}\right)\right\| u-v \|\right|, \\
\left|\left({ }^{c} D_{t}^{\gamma} T u\right)(t)-\left({ }^{c} D_{t}^{\gamma} T v\right)(t)\right| \leq & \int_{0}^{1}\left|H_{1}(t, s)\right|\left|f\left(s, u(s),{ }^{c} D_{t}^{\gamma} u(s)\right)-f\left(s, v(s),{ }^{c} D_{t}^{\gamma} v(s)\right)\right| \mathrm{d} s \\
& +\sum_{i=1}^{m}\left|H_{2}\left(t, t_{i}\right)\right|\left|J_{i}\left(u\left(t_{i}\right),{ }^{c} D_{t}^{\gamma} u\left(t_{i}\right)\right)-J_{i}\left(v\left(t_{i}\right),{ }^{c} D_{t}^{\gamma} v\left(t_{i}\right)\right)\right| \\
\leq & \int_{0}^{t}\left|H_{1}(t, s)\right|\left(a_{1}(t)+a_{2}(t)\right)\|u-v\| \mathrm{d} s+\sum_{i=1}^{m}\left|H_{2}\left(t, t_{i}\right)\right|\left(c_{1}+c_{2}\right)\|u-v\| .
\end{aligned}
$$

Thus,

$$
\begin{aligned}
\|T u-T v\| \leq & \int_{0}^{1}\left[\left|K_{1}(t, s)\right|+\left|H_{1}(t, s)\right|\right]\left(a_{1}(t)+a_{2}(t)\right)\|u-v\| \mathrm{d} s \\
& +\sum_{i=1}^{m}\left[\left|K_{2}\left(t, t_{i}\right)\right|+\left|H_{2}\left(t, t_{i}\right)\right|\right]\left(c_{1}+c_{2}\right)\|u\|+\sum_{i=1}^{m}\left\|K_{3}\left(t, t_{i}\right)\left(b_{1}+b_{2}\right)\right\| u \| \\
\leq & \Pi\|u-v\|,
\end{aligned}
$$

where $\Pi<1$, so $T$ is a contraction. Lemma 8 guarantees that $T$ has a unique fixed point in $\Omega_{R_{3}}$, which is the unique solution of (1) in $E$. This completes the proof.

\section{Examples}

In (1), let $q=1.25, \gamma=0.15, a=1, b=2, t_{1}=0.5$, and $k=1$ and then, we obtain the following fractional-order impulsive differential equation:

$$
\left\{\begin{array}{l}
{ }^{c} D_{t}^{1.25} u(t)=f\left(t, u(t),{ }^{c} D_{t}^{0.15} u(t)\right), \quad t \in(0,1), t \neq 0.5, \\
\Delta u(0.5)=I_{1}\left(u(0.5),{ }^{c} D_{t}^{0.15} u(0.5)\right), \\
\Delta^{c} D_{t}^{0.15} u(0.5)=J_{1}\left(u(0.5),{ }^{c} D_{t}^{0.15} u(0.5)\right), \\
u(0)-2 u(1)=0 \\
{ }^{c} D_{t}^{0.15} u(0)-2^{c} D_{t}^{0.15} u(1)=0 .
\end{array}\right.
$$

By a direct observation, note that $0<a<b<+\infty$, $1<q<2,0<\gamma<1$ with $q-\gamma>1$, so hypothesis (B1) is satisfied.
Example 1. In (60), let

$$
\begin{aligned}
f\left(t, u(t),{ }^{c} D_{t}^{0.15} u(t)\right) & =\frac{e^{t}}{50}+\frac{(1-t)^{2}(u(t))^{0.2}}{100}+\frac{e^{2 t}\left({ }^{c} D_{t}^{0.15} u(t)\right)^{0.3}}{200}, \\
\Delta u(0.5) & =\sin \left(\frac{1+2(u(0.5))^{0.5}+3\left({ }^{c} D_{t}^{0.15} u(0.5)\right)^{0.4}}{150}\right), \\
\Delta^{c} D_{t}^{0.25} u(0.5) & =\sin \left(\frac{1+3(u(0.5))^{0.2}+2\left({ }^{c} D_{t}^{0.15} u(0.5)\right)^{0.1}}{120}\right),
\end{aligned}
$$


so hypothesis (B2) is satisfied. Set ${ }^{c} D_{t}^{0.15} u(t)=v(t)$, and then, we obtain

$$
\begin{aligned}
& |f(t, u, v)| \leq \frac{e^{t}}{50}+\frac{(1-t)^{2}}{100}|u|^{0.2}+\frac{e^{2 t}}{200}|v|^{0.3}=a_{0}(t)+a_{1}(t)|u|^{0.2}+a_{2}(t)|v|^{0.3}, \\
& \left|I_{1}(u, v)\right| \leq \frac{1}{150}+\frac{1}{75}|u|^{0.5}+\frac{1}{50}|v|^{0.4}=b_{0}+b_{1}|u|^{0.5}+b_{2}|v|^{0.4} \\
& \left|J_{1}(u, v)\right| \leq \frac{1}{120}+\frac{1}{40}|u|^{0.2}+\frac{1}{60}|v|^{0.1}=c_{0}+c_{1}|u|^{0.2}+c_{2}|v|^{0.1}
\end{aligned}
$$

which implies that $(\mathrm{C} 1)$ and (C2) are satisfied. Thus, all the hypotheses in Theorem 1 are satisfied, so (60) has at least one solution in $E$.

Example 2. In (60), let

$$
\begin{aligned}
f\left(t, u(t),{ }^{c} D_{t}^{0.15} u(t)\right) & =\frac{(1-s)^{2}}{50} \times \frac{u(t)+{ }^{c} D_{t}^{0.15} u(t)}{1+u(t)+{ }^{c} D_{t}^{0.15} u(t)}, \\
\Delta u(0.5) & =\frac{1+2 u(0.5)+3{ }^{c} D_{t}^{0.15} u(0.5)}{150}, \\
\Delta^{c} D_{t}^{0.15} u(0.5) & =\frac{1+3 u(0.5)+2{ }^{c} D_{t}^{0.15} u(0.5)}{120},
\end{aligned}
$$

so hypothesis (B2) is satisfied. Set ${ }^{c} D_{t}^{0.15} u(t)=v(t)$, and then, we obtain

$$
\begin{aligned}
|f(t, u, v)| & \leq \frac{(1-s)^{2}}{50}=a_{0}(t), \\
\left|I_{1}\left(u_{1}, v_{1}\right)-I_{1}\left(u_{2}, v_{2}\right)\right| & \leq \frac{1}{75}\left|u_{1}-u_{2}\right|+\frac{1}{50}\left|v_{1}-v_{2}\right| \\
& =b_{1}\left|u_{1}-u_{2}\right|+b_{2}\left|v_{1}-v_{2}\right|, \\
\left|J_{1}\left(u_{1}, v_{1}\right)-J_{1}\left(u_{2}, v_{2}\right)\right| & \leq \frac{1}{40}\left|u_{1}-u_{2}\right|+\frac{1}{60}\left|v_{1}-v_{2}\right| \\
& =c_{1}\left|u_{1}-u_{2}\right|+c_{2}\left|v_{1}-v_{2}\right|,
\end{aligned}
$$

which implies that (C3) and (C4) are satisfied. Also, note that $\Lambda=0.179707<0.5$. Then, all the hypotheses in Theorem 2 are satisfied, so (60) has at least one solution in $E$.

Example 3. In (60), let

$$
\begin{aligned}
f\left(t, u(t),{ }^{c} D_{t}^{0.15} u(t)\right) & =\frac{e^{t}}{50}+\frac{(1-s)^{2} u(t)}{100}+\frac{\sqrt{(1-s)^{c}} D_{t}^{0.15} u(t)}{200}, \\
\Delta u(0.5) & =\frac{1+2 u(0.5)+3^{c} D_{t}^{0.15} u(0.5)}{150}, \\
\Delta^{c} D_{t}^{0.15} u(0.5) & =\frac{1+3 u(0.5)+2^{c} D_{t}^{0.15} u(0.5)}{120},
\end{aligned}
$$

so hypothesis (B2) is satisfied. Set ${ }^{c} D_{t}^{0.15} u(t)=v(t)$, and then, we obtain

$$
\begin{aligned}
& \left|f\left(t, u_{1}, v_{1}\right)-f\left(t, u_{2}, v_{2}\right)\right| \leq \frac{(1-s)^{2}}{100}\left|u_{1}-u_{2}\right|+\frac{\sqrt{1-s}}{200}\left|v_{1}-v_{2}\right|=a_{1}(t)\left|u_{1}-u_{2}\right|+a_{2}(t)\left|v_{1}-v_{2}\right|, \\
& \left|I_{1}\left(u_{1}, v_{1}\right)-I_{1}\left(u_{2}, v_{2}\right)\right| \leq \frac{1}{75}\left|u_{1}-u_{2}\right|+\frac{1}{50}\left|v_{1}-v_{2}\right|=b_{1}\left|u_{1}-u_{2}\right|+b_{2}\left|v_{1}-v_{2}\right| \\
& \left|J_{1}\left(u_{1}, v_{1}\right)-J_{1}\left(u_{2}, v_{2}\right)\right| \leq \frac{1}{40}\left|u_{1}-u_{2}\right|+\frac{1}{60}\left|v_{1}-v_{2}\right|=c_{1}\left|u_{1}-u_{2}\right|+c_{2}\left|v_{1}-v_{2}\right|
\end{aligned}
$$


which implies that (C4) and (C5) are satisfied. Note that $\Pi=0.787135<1$. Then, all the hypotheses in Theorem 3 are satisfied, so (60) has a unique solution in $E$.

\section{Conclusion}

In this paper, we use fixed-point theorems to study fractional-order impulsive differential equation (1) with generalized periodic boundary value conditions. Very little is known on fractional-order impulsive differential equations with generalized periodic boundary value conditions where nonlinear terms and impulse terms depend on the unknown function and the lower-order fractional derivative of the unknown function. Our main results are obtained under some nonlinear and linear growth conditions corresponding to the relevant linear operators where the symmetry property of a Green's function is not required, so our results generalize and improve works in the literature.

\section{Data Availability}

No data were used to support this study.

\section{Conflicts of Interest}

The authors declare that there are no conflicts of interest regarding the publication of this paper.

\section{Authors' Contributions}

The study was carried out in collaboration with all authors. All authors read and approved the final manuscript.

\section{Acknowledgments}

This work was supported by the University Natural Science Foundation of Anhui Provincial Education Department (Grant nos. KJ2019A0672 and KJ2018A0452), the Foundation of Suzhou University (Grant no. 2016XJGG13), the Natural Science Foundation of Chongqing (Grant no. cstc2020jcyj-msxmX0123), and the Technology Research Foundation of Chongqing Educational Committee (Grant nos. KJQN2019 00539 and KJQN202000528).

\section{References}

[1] Y. Wang and H. Wang, "Triple positive solutions for fractional differential equation boundary value problems at resonance," Applied Mathematics Letters, vol. 106, Article ID 106376, 2020.

[2] X. Hao, L. Zhang, and L. Liu, "Positive solutions of higher order fractional integral boundary value problem with a parameter," Nonlinear Analysis: Modelling and Control, vol. 24, no. 2, pp. 210-223, 2019.

[3] B. Zhu, L. Liu, and Y. Wu, "Existence and uniqueness of global mild solutions for a class of nonlinear fractional reactiondiffusion equations with delay," Computers \& Mathematics with Applications, vol. 78, no. 6, pp. 1811-1818, 2019.
[4] J. Xu, C. S. Goodrich, and Y. Cui, "Positive solutions for a system of first-order discrete fractional boundary value problems with semipositone nonlinearities," Revista de la Real Academia de Ciencias Exactas, Físicas y Naturales. Serie A. Matemáticas, vol. 113, no. 2, pp. 1343-1358, 2019.

[5] H. Zhang, Y. Li, and J. Xu, "Positive solutions for a system of fractional integral boundary value problems involving hadamard-type fractional derivatives," Complexity, vol. 2019, Article ID 2671539, 11 pages, 2019.

[6] Y. Li, J. Liu, D. O’Regan, and J. Xu, "Nontrivial solutions for a system of fractional q-difference equations involving q-integral boundary conditions," Mathematics, vol. 8, no. 5, p. 828, 2020.

[7] X. Zhang, J. Xu, J. Jiang, Y. Wu, and Y. Cui, "The convergence analysis and uniqueness of blow-up solutions for a dirichlet problem of the general k-hessian equations," Applied Mathematics Letters, vol. 102, Article ID 106124, 2020.

[8] B. Di and H. Pang, "Existence results for the fractional differential equations with multi-strip integral boundary conditions," Journal of Applied Mathematics and Computing, vol. 59, no. 1-2, pp. 1-19, 2019.

[9] Y. Niu and B. Yan, "The existence of positive solutions for the singular two-point boundary value problem," Topological Methods in Nonlinear Analysis, vol. 49, no. 2, pp. 665-682, 2017.

[10] B. Yan, "Positive solutions for the singular nonlocal boundary value problems involving nonlinear integral conditions," Boundary Value Problems, vol. 38, no. 1, 2014.

[11] S. Meng and Y. Cui, "The extremal solution to conformable fractional differential equations involving integral boundary condition,” Mathematics, vol. 7, no. 2, p. 186, 2019.

[12] Z. Ali, A. Zada, and K. Shah, "On ulam's stability for a coupled systems of nonlinear implicit fractional differential equations," Bulletin of the Malaysian Mathematical Sciences Society, vol. 42, no. 5, pp. 2681-2699, 2019.

[13] H. Zhang, Y. Li, and J. Yang, "New sequential fractional differential equations with mixed-type boundary conditions," Journal of Function Spaces, vol. 2020, p. 9, Article ID 6821637, 2020.

[14] H. Zhang, Y. Wang, and J. Xu, "Explicit monotone iterative sequences for positive solutions of a fractional differential system with coupled integral boundary conditions on a halfline," Advances in Difference Equations, vol. 2020, p. 396, 2020.

[15] S. Harendra, G. Fahimeh Akhavan, and T. Emran, "Legendre spectral method for the fractional Bratu problem," Mathematical Methods in the Applied Sciences, vol. 43, pp. 59415952, 2020.

[16] S. Harendra, G. Fahimeh Akhavan, and T. Emran, "Analysis for fractional dynamics of Ebola virus model," Chaos, Solitons \& Fractals, vol. 138, Article ID 109992, 2020.

[17] S. Harendra and H. M. Srivastava, "Numerical simulation for fractional-order bloch equation arising in nuclear magnetic resonance by using the jacobi polynomials," Applied Sciences, vol. 10, p. 285, 2020.

[18] B. Liu and Y. Liu, "Positive solutions of a two-point boundary value problem for singular fractional differential equations in banach space," Journal of Function Spaces, vol. 2013, p. 9, Article ID 585639, 2013.

[19] Y. Liu and H. Yu, "Bifurcation of positive solutions for a class of boundary value problems of fractional differential inclusions," Abstract and Applied Analysis, vol. 2013, Article ID 942831, 8 pages, 2013. 
[20] Y. Liu, "Positive solutions using bifurcation techniques for boundary value problems of fractional differential equations," Abstract and Applied Analysis, vol. 2013, Article ID 162418, 79 pages, 2013.

[21] T. Qi, Y. Liu, and Y. Cui, "Existence of solutions for a class of coupled fractional differential systems with nonlocal boundary conditions," Journal of Function Spaces, vol. 2017, Article ID 6703860, 2 pages, 2017.

[22] T. Qi, Y. Liu, and Y. Zou, "Existence result for a class of coupled fractional differential systems with integral boundary value conditions," The Journal of Nonlinear Sciences and Applications, vol. 10, no. 07, pp. 4034-4045, 2017.

[23] Y. Wang, Y. Liu, and Y. Cui, "Multiple solutions for a nonlinear fractional boundary value problem via critical point theory," Journal of Function Spaces, vol. 2017, Article ID 8548975, 8 pages, 2017.

[24] Y. Wang, Y. Liu, and Y. Cui, "Multiple sign-changing solutions for nonlinear fractional Kirchhoff equations," Boundary Value Problems, vol. 2018, p. 193, 2018.

[25] M. Kamenskii, N. Voskovskaya, and M. Zvereva, "On periodic oscillations of some points of a string with a nonlinear boundary condition," Applied Set-Valued Analysis Optimation, vol. 2, no. 1, pp. 35-48, 2020.

[26] D. Li, M. Pei, and Z. Yang, "Existence and multiplicity of positive solutions of a third-order periodic boundary value problem with one-parameter," Journal of Nonlinear Functional Analysis, vol. 2020, p. 14, 2020.

[27] A. Lachouri, A. Ardjouni, and A. Djoudi, "Positive solutions of a fractional integro-differential equation with integral boundary conditions," Communications in Optimization Theory, vol. 2020, p. 1, 2020.

[28] Y. Liu and D. O’Regan, "Controllability of impulsive functional differential systems with nonlocal conditions," Electronic Journal of Differential Equations, vol. 194, pp. 1-10, 2013.

[29] Y. Liu, "Bifurcation techniques for a class of boundary value problems of fractional impulsive differential equations," Journal of Nonlinear Sciences and Applications, vol. 09, no. 04, pp. 340-353, 2015.

[30] Y. Wang, Y. Liu, and Y. Cui, "Infinitely many solutions for impulsive fractional boundary value problem with p-laplacian," Boundary Value Problem, vol. 2018, p. 94, 2018.

[31] X. Xu, Y. Liu, H. Li, and F. E. Alsaadi, "Synchronization of switched boolean networks with impulsive effects," International Journal of Biomathematics, vol. 11, no. 06, Article ID 1850080, 2018.

[32] A. Zada, S. Fatima, Z. Ali, J. Xu, and Y. Cui, "Stability results for a coupled system of impulsive fractional differential equations," Mathematics, vol. 7, no. 10, p. 927, 2019.

[33] D. Yang, J. Wang, and D. O'Regan, “A class of nonlinear noninstantaneous impulsive differential equations involving parameters and fractional order," Applied Mathematics and Computation, vol. 321, pp. 654-671, 2018.

[34] M. Zuo, X. Hao, L. Liu, and Y. Cui, "Existence results for impulsive fractional integro-differential equation of mixed type with constant coefficient and antiperiodic boundary conditions," Boundary Value Problems, vol. 2017, p. 161, 2017.

[35] X. Liu and M. Jia, "Existence of solutions for the integral boundary value problems of fractional order impulsive differential equations," Mathematical Methods in the Applied Sciences, vol. 39, no. 3, pp. 475-487, 2016.

[36] K. Zhao and P. Gong, "Positive solutions for impulsive fractional differential equations with generalized periodic boundary value conditions," Advances in Difference Equations, vol. 2014, p. 255, 2014.

[37] Z. Bai, X. Dong, and C. Yin, "Existence results for impulsive nonlinear fractional differential equation with mixed boundary conditions," Boundary Value Problems, vol. 201663 pages, 2016.

[38] K. Zhang and J. Xu, "Positive solutions for an impulsive boundary value problem with Caputo fractional derivative," Journal of Nonlinear Sciences and Applications, vol. 09, no. 06, pp. 4628-4638, 2016.

[39] I. Podlubny, Fractional Differential Equations, Academic Press, New York, NY, USA,, 1993.

[40] A. A. Kilbas, H. M. Srivastava, and J. J. Trujillo, Theory and Applications of Fractional Differential Equations, NorthHolland Mathematics Studies. Elsevier, Amsterdam, Netherlands, 2006.

[41] K. Deimling, Nonlinear Functional Analysis, Spring, Berlin, Germany, 1985.

[42] M. A. Krasnoselskii, Topological Methods in the Theory of Nonlinear Integral Equations, Pergamon Press, New York, NY, USA, 1964.

[43] A. Granas and J. Dugundji, Fixed Point Theory, Spring, New York, NY, USA, 2003. 